\title{
Abdominal free fluid in acute pancreatitis predicts necrotizing pancreatitis and organ failure
}

\author{
Edward Yang, Nghia H. Nguyen, Wilson T. Kwong \\ UC San Diego Division of Gastroenterology, Department of Medicine, La Jolla, California, USA
}

\section{Abstract}

\section{Introduction}

The clinical course of acute pancreatitis ranges from a mild, self-limited illness to a fulminant, fatal course and the type can be indistinguishable upon initial presentation. Predicting the clinical course in acute pancreatitis remains challenging, despite decades of studies investigating a wide variety of clinical, imaging, and serological predictors.

Division of Gastroenterology, Department of Medicine, UC San Diego, La Jolla, California, USA

Conflict of Interest: None

Correspondence to: Wilson T. Kwong, 200 W. Arbor Drive \#8413, San

Diego, CA 92103, USA, e-mail: wtkwong@health.ucsd.edu

Received 29 March 2021; accepted 7 June 2021;

published online 14 September 2021

DOI: https://doi.org/10.20524/aog.2021.0666
Cross-sectional abdominal imaging, mainly computed tomography (CT) scanning, is performed in $60-70 \%$ patients with acute pancreatitis within the first $24-48 \mathrm{~h}$ of presentation $[1,2]$. While the intent of the imaging study is primarily diagnostic, there is much information from cross-sectional imaging that may be harnessed for predictive purposes, especially with the emergence of artificial neural networks. Traditional predictors for severity in acute pancreatitis, including persistent systemic inflammatory response syndrome (SIRS), blood urea nitrogen (BUN), hematocrit (HCT), Ranson criteria, and other clinical scoring systems, have only been proven to be moderately effective and can take up to $48 \mathrm{~h}$ to calculate or measure [3-8]. CT imaging features have the benefit of immediate acquisition and the examination is already performed in the majority of patients.

Free abdominal fluid, or ascites, is often observed in acute pancreatitis, but this finding is frequently overlooked, underreported in some radiologic interpretations, or not considered to be of clinical significance unless large in volume or creating 
increased abdominal pressure. The release of free fluid in acute pancreatitis may be due to either pancreatic duct disruption, possibly from developing pancreatic necrosis, or capillary leakage resulting from elevated inflammatory mediators. In both instances, the course of pancreatitis is potentially more severe. A recent study examining 82 patients with pancreatitis and ascites on imaging reported longer hospital stays, and higher rates of organ failure and mortality, compared to pancreatitis patients without ascites on imaging [9].

Based on our own clinical experience with pancreatitis patients, we hypothesized that any pancreatitis-related free fluid detected on cross-sectional imaging, even small volumes that might not be sufficient to be categorized as ascites, could be a sign of increased severity. We carried out the following study to assess the utility of pancreatitis-related free fluid as a predictor for the development of necrosis as well as a complicated clinical course. If free fluid can portend the development of necrosis, this could be useful, as pancreatic necrosis can take up to 3-4 days to become evident on imaging and requires intravenous contrast for detection. Free abdominal fluid may precede the presence of overt necrosis and can be readily identified on imaging, even in the absence of intravenous contrast administration.

\section{Patients and methods}

This was a single-center retrospective clinical study, designed to assess whether free abdominal fluid in acute pancreatitis can predict the subsequent development of pancreatic necrosis, as well as other adverse outcomes including organ failure and mortality.

\section{Patient population}

Data were extracted from electronic medical records at the University of California San Diego Medical Center (La Jolla, CA). Patients receiving medical care between January 1, 2008, and December 31, 2019, were considered for this study. The study included patients at least 18 years of age, diagnosed with acute pancreatitis, who had at least 2 cross-sectional imaging studies within 3 months of acute pancreatitis diagnosis. A diagnosis of acute pancreatitis required the presence of 2 of the following: lipase elevation greater than 3 times the upper limit of normal, imaging evidence of pancreatitis, or characteristic abdominal pain. Subjects in this study were required to have an initial contrast-enhanced cross-sectional imaging study (CT or magnetic resonance imaging [MRI]) without pancreatic necrosis, and a subsequent cross-sectional imaging study within 3 months to reassess for the development of pancreatic necrosis. The indication for the subsequent imaging study could be pancreatitis-related, for a biliary indication, or for an unrelated indication.

Excluded were patients with pancreatic necrosis evident on the initial imaging study, pre-existing ascites, risk factors for ascites (cirrhosis, prior cardiac ascites, peritoneal dialysis, recent abdominal surgery, gastrointestinal perforation, or peritoneal carcinomatosis), pancreatic cancer, chronic pancreatitis, and recurrent pancreatitis with a known unresolved fluid collection.

\section{Study definitions}

Necrotizing pancreatitis was diagnosed based on its typical appearance on contrast-enhanced CT and MRI scans. Organ failure was based on the Modified Marshall Score as cardiovascular, respiratory or renal failure. Transient organ failure was defined as organ failure lasting $<48 \mathrm{~h}$, and persistent organ failure as lasting $>48 \mathrm{~h}$. BUN elevation was defined as BUN $>20 \mathrm{mg} / \mathrm{dL}$ on admission or as an increase by $>5 \mathrm{mg} / \mathrm{dL}$ at $24 \mathrm{~h}$. HCT elevation was defined as HCT $>44 \%$ or failure to decrease at $24 \mathrm{~h}$. Free fluid was defined as free-flowing fluid in the peritoneal cavity or ascites. For the purposes of this study, free fluid was attributed to the current episode of acute pancreatitis. Those with previous ascites from other causes were excluded. When there was uncertainty regarding the presence of acute free fluid, a gastroenterologist with extensive training in advanced endoscopic imaging and pancreatology with more than 5 years in practice reviewed the imaging study while blinded to the clinical outcome.

\section{Outcomes and predictors}

Free fluid was assessed along with commonly utilized predictors of pancreatitis severity (persistent SIRS, BUN elevation at $24 \mathrm{~h}$, and hemoconcentration on HCT at $24 \mathrm{~h}$ ). The primary outcome was the presence of necrotizing pancreatitis, defined as pancreatic parenchymal necrosis, peri-pancreatic necrosis, acute necrotic collection or walled-off necrosis. Secondary outcomes were mortality (6-month and inhospital), transient organ failure, persistent organ failure, and a composite endpoint composed of necrotizing pancreatitis, persistent organ failure, or 6-month mortality attributed to complications of acute pancreatitis.

\section{Statistical analysis}

Descriptive statistical tests were performed to describe the characteristics of our cohort. To compare differences in baseline characteristics and treatment outcomes, we used the chi-squared test to analyze categorical variables and a $t$-test to analyze continuous variables. To identify predictive factors associated with our outcome, we used binomial logistic regression to identify factors associated with the development of pancreatic necrosis and a composite outcome of pancreatic necrosis, persistent organ failure or 6-month mortality. A univariate analysis was first performed to identify factors (based on a P-value cut-off of $<0.20$ ) that would be appropriate for inclusion in our final multivariable model. Our multivariable logistic regression model was used to calculate the area under 
the receiver operator curve (AUC). All hypothesis testing was performed using a 2-sided $\mathrm{P}$-value with a statistical significance threshold $<0.05$. All statistical analyses were performed using StataMP (StataCorp. 2015. Stata Statistical Software: Release 14. College Station, TX: StataCorp LP).

\section{Ethics}

The study protocol and methods were approved by the institutional review board at the University of California, San Diego. All authors had access to the study data and reviewed and approved of the final manuscript.

\section{Results}

\section{Patient and imaging characteristics}

A total of 245 patients with acute pancreatitis meeting the imaging criteria were included in this study. There was no significant difference in age or sex between pancreatitis patients with free fluid or no free fluid on initial imaging (Table 1). Nor was there any significant difference in lipase elevation ( 85.4 vs. $81.9 \%, \mathrm{P}=0.469$ ), $\mathrm{BUN}$ value on admission (17.0 \pm 15.9 vs. $14.9 \pm 8.7 \mathrm{mg} / \mathrm{dL}, \mathrm{P}=0.186), \mathrm{BUN}$ value at $24 \mathrm{~h}$ $(14.7 \pm 16.3$ vs. $12.0 \pm 8.0 \mathrm{mg} / \mathrm{dL}, \mathrm{P}=0.122)$, HCT value on admission ( $37.5 \pm 8.9$ vs. $37.8 \pm 5.6 \%, \mathrm{P}=0.786)$, or $\mathrm{HCT}$ value at $24 \mathrm{~h}$ ( $35.9 \pm 7.3$ vs. $35.2 \pm 4.8 \%, \mathrm{P}=0.443)$, respectively. In both groups, the most common indication for obtaining the initial

Table 1 Patient characteristics

\begin{tabular}{|c|c|c|c|c|}
\hline Characteristics & $\begin{array}{l}\text { Fluid } \\
\mathrm{n}=96\end{array}$ & $\begin{array}{l}\text { No fluid } \\
\mathrm{n}=149\end{array}$ & $\mathrm{P}$-value & $\begin{array}{l}\text { Entire } \\
\text { cohort } \\
n=245\end{array}$ \\
\hline $\begin{array}{l}\text { Age (years, } \\
\text { mean } \pm S D)\end{array}$ & $48.4 \pm 16.8$ & $52.7 \pm 16.5$ & 0.050 & $51.0 \pm 16.7$ \\
\hline \multicolumn{5}{|l|}{ Sex } \\
\hline Male & $54.2 \%$ & $48.6 \%$ & 0.319 & $50.2 \%$ \\
\hline Female & $45.8 \%$ & $52.4 \%$ & & $49.8 \%$ \\
\hline \multicolumn{5}{|l|}{ Alcohol use } \\
\hline Yes & $24.0 \%$ & $15.4 \%$ & 0.148 & $18.8 \%$ \\
\hline None & $75.0 \%$ & $81.2 \%$ & & $78.8 \%$ \\
\hline Not stated & $1.0 \%$ & $3.4 \%$ & & $2.5 \%$ \\
\hline \multicolumn{5}{|l|}{ Smoking } \\
\hline Active & $16.7 \%$ & $16.1 \%$ & 0.351 & $16.3 \%$ \\
\hline Former & $12.5 \%$ & $21.5 \%$ & & $18.0 \%$ \\
\hline None & $63.5 \%$ & $57.9 \%$ & & $59.2 \%$ \\
\hline Not stated & $7.3 \%$ & $6.0 \%$ & & $6.5 \%$ \\
\hline \multicolumn{5}{|l|}{ Etiology } \\
\hline Gallstones & $29.2 \%$ & $26.9 \%$ & 0.034 & $27.8 \%$ \\
\hline Alcohol & $18.8 \%$ & $12.1 \%$ & & $14.7 \%$ \\
\hline $\begin{array}{l}\text { Idiopathic / } \\
\text { other }\end{array}$ & $47.9 \%$ & $45.6 \%$ & & $46.5 \%$ \\
\hline Not stated & $4.2 \%$ & $15.4 \%$ & & $11.0 \%$ \\
\hline
\end{tabular}

imaging study was pancreatitis-related $(99.0 \%$ in free fluid group vs. $98.0 \%$ in no free fluid group), and the predominant reason for obtaining a subsequent imaging study was for pancreatitis-related and biliary indications. The initial crosssectional imaging study was obtained within 2 days of the acute pancreatitis diagnosis in $96.7 \%$ of patients, with $76.7 \%$ of imaging studies obtained at the time of diagnosis.

\section{Pancreatitis outcomes based on presence of fluid}

In the entire cohort, $13.1 \%(n=32)$ of patients developed pancreatic necrosis. Transient organ failure, persistent organ failure, in-hospital mortality and 6-month mortality occurred in $9.0 \%(n=22), 8.2 \%(n=20), 3.7 \%(n=9)$, and $5.3 \%(n=13)$ of the entire study population, respectively.

The primary endpoint of pancreatic necrosis occurred in $31.3 \%(n=30)$ of patients with acute pancreatitis and free fluid on the initial imaging study, compared to $1.3 \%(\mathrm{n}=2)$ in patients without free fluid $(\mathrm{P}<0.001)$. Infected necrosis occurred more frequently in the fluid group (Table 2). More than twice the number of patients with fluid had HCT elevation, but there was no difference in BUN elevation (Table 2). Compared to patients without free fluid on the initial imaging study, those with free fluid had higher rates of transient organ failure (17.7 vs. $3.4 \%, \mathrm{P}<0.001)$, persistent organ failure (17.7 vs. $2.0 \%, \mathrm{P}<0.001)$, 6-month mortality (9.4 vs. $2.7 \%, \mathrm{P}=0.023$ ), and in-hospital mortality (7.3 vs. $1.3 \%, \mathrm{P}=0.016$ ) (Table 2 ). The composite endpoint of pancreatic necrosis, 6-month mortality or persistent organ failure occurred in $37.5 \%$ of patients with fluid on initial imaging compared to $4.7 \%$ of those with no fluid $(\mathrm{P}<0.001)$.

An invasive procedure for drainage was required in $13.5 \%$ $(n=13)$ of patients with acute pancreatitis and free fluid on initial imaging compared to $0.5 \%(n=1)$ in those without free fluid (Table 2). The total hospital stay in patients with free fluid on initial imaging was nearly 3 times longer than in those without free fluid $(16.2 \pm 24.0$ days, $95 \%$ confidence interval [CI] 11.3-21.0, vs. 5.5 \pm 7.8 days, 95\%CI 4.3-6.8; $\mathrm{P}<0.001$ ), and transfer to the intensive care unit (ICU) occurred more frequently ( 30.2 vs. $4.7 \%, \mathrm{P}<0.001$ ). There was no difference in 30-day readmission rates between fluid groups (Table 2).

Pancreatitis outcomes were also assessed for the entire cohort based on the development of necrosis, regardless of fluid status. Patients who eventually developed necrotizing pancreatitis had higher rates of transient organ failure (37.5 vs. 4.7\%, $\mathrm{P}<0.001)$, persistent organ failure (37.5 vs. $3.8 \%$, $\mathrm{P}<0.001$ ), 6-month mortality (15.6 vs. $3.8 \%, \mathrm{P}=0.004)$ and in-hospital mortality ( 12.5 vs. $2.35 \%, \mathrm{P}=0.005)$. Patients with necrosis also had longer hospital stays and higher rates of ICU admission (Supplementary Table 1).

\section{Predicting pancreatic necrosis and pancreatitis outcomes}

We compared the performance of free fluid with persistent SIRS, BUN and HCT concentrations on admission, BUN 
Table 2 Primary and secondary pancreatitis outcomes based on fluid or no fluid on imaging

\begin{tabular}{|c|c|c|c|c|c|c|c|}
\hline \multirow{2}{*}{$\begin{array}{l}\text { Outcomes } \\
\text { Necrosis }\end{array}$} & \multicolumn{2}{|c|}{ Fluid $n=96$} & \multicolumn{2}{|c|}{ No fluid $n=149$} & \multirow{2}{*}{$\begin{array}{c}\text { P-value } \\
<0.001\end{array}$} & \multicolumn{2}{|c|}{ Entire cohort $n=245$} \\
\hline & $31.3 \%$ & $\mathrm{n}=30$ & $1.3 \%$ & $\mathrm{n}=2$ & & $13.1 \%$ & $\mathrm{n}=32$ \\
\hline Extra-pancreatic & $2.1 \%$ & $\mathrm{n}=2$ & $0.7 \%$ & $\mathrm{n}=1$ & - & $1.2 \%$ & $\mathrm{n}=3$ \\
\hline Infected necrosis & $5.2 \%$ & $\mathrm{n}=5$ & $0.0 \%$ & $\mathrm{n}=0$ & 0.005 & $2.0 \%$ & $\mathrm{n}=5$ \\
\hline Composite endpoint $^{\dagger}$ & $37.5 \%$ & $\mathrm{n}=36$ & $4.7 \%$ & $\mathrm{n}=7$ & $<0.001$ & $17.6 \%$ & $\mathrm{n}=43$ \\
\hline \multicolumn{8}{|l|}{ Mortality } \\
\hline Six-month & $7.3 \%$ & $\mathrm{n}=7$ & $1.3 \%$ & $\mathrm{n}=2$ & 0.016 & $3.7 \%$ & $\mathrm{n}=9$ \\
\hline In-hospital & $9.4 \%$ & $\mathrm{n}=9$ & $2.7 \%$ & $\mathrm{n}=4$ & 0.023 & $5.3 \%$ & $\mathrm{n}=13$ \\
\hline Transient organ failure ${ }^{\ddagger}$ & $17.7 \%$ & $\mathrm{n}=17$ & $3.4 \%$ & $\mathrm{n}=5$ & $<0.001$ & $9.0 \%$ & $\mathrm{n}=22$ \\
\hline Cardiac & $9.4 \%$ & $\mathrm{n}=9$ & $1.3 \%$ & $\mathrm{n}=2$ & 0.003 & $4.5 \%$ & $\mathrm{n}=11$ \\
\hline Respiratory & $12.5 \%$ & $\mathrm{n}=12$ & $3.4 \%$ & $\mathrm{n}=5$ & 0.006 & $6.9 \%$ & $\mathrm{n}=17$ \\
\hline Renal & $10.4 \%$ & $\mathrm{n}=10$ & $1.3 \%$ & $\mathrm{n}=2$ & 0.001 & $4.9 \%$ & $\mathrm{n}=12$ \\
\hline Persistent organ failure ${ }^{\ddagger}$ & $17.7 \%$ & $\mathrm{n}=17$ & $2.0 \%$ & $\mathrm{n}=3$ & $<0.001$ & $8.2 \%$ & $\mathrm{n}=20$ \\
\hline Cardiac & $10.4 \%$ & $\mathrm{n}=10$ & $0.7 \%$ & $\mathrm{n}=1$ & $<0.001$ & $4.5 \%$ & $\mathrm{n}=11$ \\
\hline Respiratory & $12.5 \%$ & $\mathrm{n}=12$ & $2.0 \%$ & $\mathrm{n}=3$ & 0.001 & $6.1 \%$ & $\mathrm{n}=15$ \\
\hline Renal & $12.5 \%$ & $\mathrm{n}=12$ & $0.7 \%$ & $\mathrm{n}=1$ & $<0.001$ & $5.3 \%$ & $\mathrm{n}=13$ \\
\hline BUN elevation & $22.9 \%$ & $\mathrm{n}=22$ & $22.8 \%$ & $\mathrm{n}=34$ & 0.986 & $22.9 \%$ & $\mathrm{n}=56$ \\
\hline Hematocrit elevation & $46.9 \%$ & $\mathrm{n}=45$ & $21.5 \%$ & $\mathrm{n}=32$ & $<0.001$ & $31.4 \%$ & $\mathrm{n}=77$ \\
\hline Drainage procedure & $13.5 \%$ & $\mathrm{n}=13$ & $0.5 \%$ & $\mathrm{n}=1$ & 0.009 & $5.7 \%$ & $\mathrm{n}=14$ \\
\hline Endoscopic & $4.2 \%$ & $\mathrm{n}=4$ & $0.7 \%$ & $\mathrm{n}=1$ & & $2.0 \%$ & $\mathrm{n}=5$ \\
\hline Radiologic & $5.2 \%$ & $\mathrm{n}=5$ & $0.0 \%$ & $\mathrm{n}=0$ & & $2.0 \%$ & $\mathrm{n}=5$ \\
\hline Surgical & $4.2 \%$ & $\mathrm{n}=4$ & $0.0 \%$ & $\mathrm{n}=0$ & & $1.6 \%$ & $\mathrm{n}=4$ \\
\hline Length of hospital stay (mean $\pm S D)$ & \multicolumn{2}{|c|}{$16.2 \pm 24.0$ days } & \multicolumn{2}{|c|}{$5.5 \pm 7.8$ days } & & \multicolumn{2}{|c|}{$9.7 \pm 17.0$ days } \\
\hline ICU admission & $30.2 \%$ & $\mathrm{n}=29$ & $4.7 \%$ & $\mathrm{n}=7$ & $<0.001$ & $14.7 \%$ & $\mathrm{n}=36$ \\
\hline ER discharge ${ }^{\S}$ & $3.1 \%$ & $\mathrm{n}=3$ & $20.1 \%$ & $\mathrm{n}=30$ & $<0.001$ & $13.5 \%$ & $\mathrm{n}=33$ \\
\hline Outpatient & $1.0 \%$ & $\mathrm{n}=1$ & $4.7 \%$ & $\mathrm{n}=7$ & $<0.001$ & $3.3 \%$ & $\mathrm{n}=8$ \\
\hline 30-day readmission & $17.7 \%$ & $\mathrm{n}=17$ & $20.1 \%$ & $\mathrm{n}=30$ & 0.638 & $19.2 \%$ & $\mathrm{n}=47$ \\
\hline
\end{tabular}

${ }^{\dagger}$ Composite endpoint: development of either pancreatic necrosis, persistent organ failure, or death at 6 months

${ }^{\ddagger}$ Organ failure is defined as failure of one or more organ systems, with failure lasting $>48 \mathrm{~h}$ considered persistent organ failure

${ }^{\S}$ Emergency room (ER) discharge indicates patients presenting to the ER with interstitial edematous pancreatitis but never required admission

BUN, blood urea nitrogen; SD, standard deviation

elevation, and HCT elevation, regarding their ability to predict the outcomes of pancreatic necrosis and a composite endpoint of pancreatic necrosis, persistent organ failure or mortality. Univariate logistic regression analysis revealed that free fluid had the highest odds for predicting pancreatic necrosis (odds ratio [OR] 33.41, 95\%CI 7.75-143.93; $\mathrm{P}<0.001)$ followed by persistent SIRS (OR 20.20, 95\%CI 8.06-50.62; $\mathrm{P}<0.001$ ); BUN and HCT elevation had only modest predictive value and BUN or HCT values alone were not predictive (Table 3 ). In predicting the composite endpoint, persistent SIRS demonstrated slightly better performance (OR 17.45, 95\%CI 7.99-38.28; $\mathrm{P}<0.001$ ) compared to free fluid (OR 12.17, 95\%CI 5.13-28.90; $\mathrm{P}<0.001$ ), while BUN or HCT elevations were also predictive, though not as strongly (Table 3).

On multivariate logistic regression, free fluid was the strongest predictor (adjusted OR 17.11, 95\%CI 3.68-79.65; $\mathrm{P}<0.001)$ for the development of necrotizing pancreatitis followed by persistent SIRS (adjusted OR 7.70, 95\%CI 2.7221.82; $\mathrm{P}<0.001$ ); HCT elevation on admission was only modestly predictive and BUN elevation was not significant (Table 3). This model performed very well in predicting necrotizing pancreatitis, with an AUC of 0.92. For the composite endpoint, multivariate logistic regression showed similar performance between free fluid (adjusted OR 7.49, 95\%CI 2.76-20.35; $\mathrm{P}<0.001$ ) and persistent SIRS (adjusted OR 7.12, 95\%CI 2.98-17.22; $\mathrm{P}<0.001$ ), with only modest predictive value for BUN elevation (Table 3 ).

Compared to persistent SIRS, the presence of free fluid on initial cross-sectional imaging in patients with acute pancreatitis demonstrated a higher sensitivity $(93.8 \%, 95 \% \mathrm{CI}$ 79.2-99.2\% vs. $78.1 \%, 95 \%$ CI $60.0-90.7 \%$ ) but lower specificity (69.0\%, 95\%CI $62.3-75.2 \%$ vs. $85.0 \%$, 95\%CI 79.5-89.5\%), for development of pancreatic necrosis (Table 4). The absence of free fluid on the initial imaging study demonstrated a better negative predictive value compared to persistent SIRS, though both predictors had only modest positive predictive value (Table 4). The presence of free fluid was also more sensitive than persistent SIRS for developing the composite endpoint $(83.7 \%$, 95\%CI $69.3-93.2 \%$ vs. $72.1 \%$, 95\%CI 56.3 $84.7 \%$ ), while both predictors showed similar sensitivities for developing persistent organ failure or death at 6 months (Table 4). 
Table 3 Single and multiple logistic regressions for predictors of pancreatic necrosis and the composite endpoints

\begin{tabular}{|c|c|c|c|c|}
\hline Predictors & Odds ratio $(95 \% \mathrm{CI})$ & P-value & Adjusted odds ratio (95\%CI) & P-value \\
\hline \multicolumn{5}{|l|}{ Pancreatic necrosis } \\
\hline Free fluid & $33.41(7.75-143.93)$ & $<0.001$ & $17.11(3.68-79.65)$ & $<0.001$ \\
\hline Persistent SIRS & $20.20(8.06-50.62)$ & $<0.001$ & $7.70(2.72-21.82)$ & $<0.001$ \\
\hline BUN level (admit) $)^{\dagger}$ & $1.02(0.99-1.04)$ & 0.168 & -- & --- \\
\hline BUN elevation & $2.31(1.05-5.07)$ & 0.038 & $1.68(0.57-5.00)$ & 0.350 \\
\hline Hematocrit level (admit) ${ }^{\dagger}$ & $1.05(0.99-1.12)$ & 0.093 & --- & --- \\
\hline Hematocrit elevation (admit) $)^{\S}$ & $4.40(2.00-9.72)$ & $<0.001$ & $2.80(1.01-7.79)$ & 0.048 \\
\hline Hematocrit elevation ${ }^{*}$ & $6.32(2.82-14.18)$ & $<0.001$ & --- & --- \\
\hline \multicolumn{5}{|l|}{ Composite endpoint } \\
\hline Free fluid & $12.17(5.13-28.90)$ & $<0.001$ & $7.49(2.76-20.35)$ & $<0.001$ \\
\hline Persistent SIRS & $17.45(7.99-38.28)$ & $<0.001$ & $7.12(2.98-17.22)$ & $<0.001$ \\
\hline BUN level (admit) ${ }^{\dagger}$ & $1.05(1.02-1.08)$ & 0.001 & -- & --- \\
\hline BUN elevation ${ }^{*}$ & $3.53(1.75-7.12)$ & $<0.001$ & $3.40(1.31-8.81)$ & 0.012 \\
\hline Hematocrit level (admit) ${ }^{\dagger}$ & $0.99(0.95-1.04)$ & 0.780 & -- & --- \\
\hline Hematocrit elevation (admit) ${ }^{\S}$ & $2.96(1.42-6.16)$ & 0.004 & $1.74(0.67-4.50)$ & 0.252 \\
\hline Hematocrit elevation ${ }^{*}$ & $3.55(1.80-7.00)$ & $<0.001$ & --- & --- \\
\hline
\end{tabular}

${ }^{\dagger}$ Actual lab value of BUN or hematocrit on admission

${ }^{\ddagger}$ BUN elevation defined as BUN $>20 \mathrm{mg} / \mathrm{dL}$ on admission or increase by $>5 \mathrm{mg} / \mathrm{dL}$

${ }^{\varsigma}$ Hematocrit elevation on admission defined as value $>44 \%$

"Hematocrit elevation overall defined as value $>44 \%$ on admission or failure to decrease at $24 \mathrm{~h}$

SIRS, systemic inflammatory response syndrome; BUN, blood urea nitrogen

Table 4 Fluid and persistent SIRS as predictors of pancreatic necrosis and pancreatitis outcomes

\begin{tabular}{|c|c|c|c|c|c|}
\hline Predictors & Sensitivity & Specificity & PPV & NPV & Accuracy \\
\hline \multicolumn{6}{|l|}{ Free fluid as a predictor of } \\
\hline Pancreatic necrosis & $93.8 \%$ & $69.0 \%$ & $31.3 \%$ & $98.7 \%$ & $72.2 \%$ \\
\hline Persistent organ failure & $85.0 \%$ & $64.9 \%$ & $17.7 \%$ & $98.0 \%$ & $66.5 \%$ \\
\hline Six-month mortality & $69.2 \%$ & $62.5 \%$ & $9.4 \%$ & $97.3 \%$ & $62.9 \%$ \\
\hline Composite endpoint ${ }^{*}$ & $83.7 \%$ & $70.3 \%$ & $37.5 \%$ & $95.3 \%$ & $72.7 \%$ \\
\hline \multicolumn{6}{|c|}{ Persistent SIRS as a predictor of } \\
\hline Pancreatic necrosis & $78.1 \%$ & $85.0 \%$ & $43.9 \%$ & $96.3 \%$ & $84.1 \%$ \\
\hline Persistent organ failure & $90.0 \%$ & $82.7 \%$ & $31.6 \%$ & $98.9 \%$ & $83.3 \%$ \\
\hline Six-month mortality & $69.2 \%$ & $79.3 \%$ & $15.8 \%$ & $97.9 \%$ & $78.8 \%$ \\
\hline Composite endpoint ${ }^{*}$ & $72.1 \%$ & $87.2 \%$ & $54.4 \%$ & $93.6 \%$ & $84.5 \%$ \\
\hline
\end{tabular}

"Composite endpoint: development of either pancreatic necrosis, persistent organ failure, or death at 6 months

PPV, positive predictive value; NPV, negative predictive value; SIRS, systemic inflammatory response syndrome

When neither fluid on initial imaging nor persistent SIRS were present, the negative predictive values for developing pancreatic necrosis, persistent organ failure, 6-month mortality and the composite endpoint were 100\% (95\% CI: 100\%), 99.3\% (95\% CI: 95.2-99.9\%), 97.8\% (95\% CI: 94.2-99.2\%), and 97.8\% (95\% CI: 93.7-99.3\%), respectively.

\section{Discussion}

The current study examined a novel imaging-based predictor of free fluid for adverse outcomes of necrotizing pancreatitis, organ failure, and mortality. Not all patients presenting with acute pancreatitis require a CT scan, but approximately $70 \%$ already undergo a CT scan within 24-48 h of presentation, usually because of diagnostic uncertainty or to assess for complications. Given that cross- sectional imaging is available in most cases, we should maximize the information that can be extracted from the images. Several previous studies have utilized imaging for various purposes in acute pancreatitis. The modified CT severity index provides a scoring system, including necrosis, inflammation and ascites, to classify the severity of pancreatitis. Perfusion CT has been used to identify ischemia and predict necrosis, with moderate performance. Artificial neural networks have also been studied in the prediction of pancreatitis outcomes. Without the necessity for special imaging protocols, software or expertise, abdominal free fluid can be identified easily, even in the absence of intravenous contrast.

Samanta et al examined 82 patients with ascites, defined as free-flowing peritoneal fluid at least $50 \mathrm{~mL}$ in volume [9]. The presence of pancreatitis-related ascites was associated with a greater length of stay, need for ICU admission, organ failure, and mortality. The presence of ascites was assessed 
within 2 weeks of the manifestation of acute pancreatitis, which did not allow its assessment as a potential predictor for complications of acute pancreatitis.

Our clinical experience in managing patients with necrotizing pancreatitis led us to carry out the current study, which examined the presence of any amount of free fluid in the abdomen in relation to pancreatitis, as a predictor of necrosis and other adverse outcomes. We hypothesized that the presence of free fluid would correlate most closely with the local complication of necrosis. Sufficient injury to the pancreatic ductal system to facilitate free fluid leakage into the abdomen may be a subtle or early sign of necrosis. Alternatively, free fluid related to capillary leak, as a result of increased inflammation and cytokine release, could also be indicative of occult or developing necrosis driving a proinflammatory response.

The current multivariate logistic regression analysis demonstrated pancreatitis-related free fluid was the strongest predictor of necrosis, and of a composite endpoint including organ failure and mortality. Free fluid outperformed persistent SIRS, BUN and HCT as a predictor, with a high AUC of 0.92 indicating excellent performance in classifying and predicting outcomes. Free fluid demonstrates a high sensitivity of $93.8 \%$ for the subsequent development of necrosis, which is desirable for a screening test for necrosis. The tradeoff is a moderate positive predictive value of $31.3 \%$, indicating that about 1 in 3 patients with free fluid will go on to develop necrosis if not already apparent on imaging. In this study, we excluded patients who already had necrosis apparent on initial imaging so that free fluid could be studied as a predictive factor for occult or developing necrosis.

In addition, pancreatitis-related free fluid demonstrates a high negative predictive value of $98.7 \%$ for necrosis and $95.3 \%$ for the composite endpoint including mortality and organ failure. Therefore, the absence of free fluid on cross-sectional imaging during acute pancreatitis is very reassuring and may also be utilized as a factor in the early discharge of pancreatitis patients or as a triage tool in the emergency room. Notably, no patients lacking both free fluid and persistent SIRS developed pancreatic necrosis and less than $1 \%$ developed persistent organ failure, suggesting that the absence of both these predictors is highly predictive of a benign course: this may be the most useful of the findings of this study. Future studies will be necessary to confirm and validate the findings of the current study, but free fluid appears to be a strong and promising marker to predict developing necrosis, while also reassuring those who are likely to have a benign course.

The current study findings have to be evaluated with some caveats. A potential source of bias in the current study was the requirement for patients to have 2 cross-sectional imaging studies for inclusion, which introduces a potential bias towards patients who are more ill, with higher rates of necrosis or organ failure, and may have missed milder cases of pancreatitis. However, our rates of necrosis, persistent organ failure and mortality are consistent with other larger studies that did not have this inclusion criterion. The study also had only a moderate sample size, and a larger or multicenter sample could further reinforce our findings. The adjusted OR for free fluid as a predictor of necrosis, organ failure and mortality was much higher than that for established predictors such as persistent SIRS and BUN, which may suggest that the finding will remain significant with more data. Future prospective studies on the predictive ability of fluid on cross-sectional imaging are needed to support our findings and should include all severities of acute pancreatitis.

In conclusion, we report that free fluid on imaging in acute pancreatitis predicts the development of necrotizing pancreatitis, as well as organ failure and mortality. Free fluid performed better than traditional predictors in this capacity. The absence of free fluid was highly reassuring, especially in the absence of persistent SIRS: in this scenario, patients did not develop necrosis and rarely developed organ failure. These low-risk patients may be considered for early discharge.

\section{Summary Box}

\section{What is already known:}

- The clinical course of acute pancreatitis varies from mild, self-limited disease to a fulminant, fatal course

- Predicting pancreatitis severity remains challenging, despite decades of studies on clinical, imaging, and serological predictors

- Cross-sectional imaging is available in the majority of patients with acute pancreatitis and contains much information that is potentially underutilized.

- Free abdominal fluid is a radiologic finding in acute pancreatitis that is frequently overlooked

\section{What the new findings are:}

- Free fluid on imaging for acute pancreatitis is a strong predictor for subsequent development of necrotizing pancreatitis

- The presence of fluid can predict adverse outcomes, including organ failure and mortality, and outperformed persistent systemic inflammatory response syndrome (SIRS), blood urea nitrogen, and hematocrit elevation in predicting these outcomes

- In patients with acute pancreatitis, available imaging studies should be reviewed to assess for the presence of free fluid, as this can identify patients who should be closely monitored for severe outcomes

- Patients who lack free fluid on imaging and also do not demonstrate SIRS have very low risk for adverse outcomes and may be considered for early discharge 


\section{References}

1. Dachs RJ, Sullivan L, Shanmugathasan P. Does early ED CT scanning of afebrile patients with first episodes of acute pancreatitis ever change management? Emerg Radiol 2015;22:239-243.

2. Shinagare AB, Ip IK, Raja AS, Sahni VA, Banks P, Khorasani R. Use of CT and MRI in emergency department patients with acute pancreatitis. Abdom Imaging 2015;40:272-277.

3. Koutroumpakis E, Wu BU, Bakker OJ, et al. Admission hematocrit and rise in blood urea nitrogen at $24 \mathrm{~h}$ outperform other laboratory markers in predicting persistent organ failure and pancreatic necrosis in acute pancreatitis: a post hoc analysis of three large prospective databases. Am J Gastroenterol 2015;110:1707-1716.

4. Mounzer R, Langmead CJ, Wu BU et al. Comparison of existing clinical scoring systems to predict persistent organ failure in patients with acute pancreatitis. Gastroenterology 2012;142:
1476-1482.

5. Wu BU, Bakker OJ, Papachristou GI, et al. Blood urea nitrogen in the early assessment of acute pancreatitis: an international validation study. Arch Intern Med 2011;171:669-676.

6. Singh $\mathrm{VK}, \mathrm{Wu} \mathrm{BU}$, Bollen $\mathrm{TL}$, et al. A prospective evaluation of the bedside index for severity in acute pancreatitis score in assessing mortality and intermediate markers of severity in acute pancreatitis. Am J Gastroenterol 2009;104:966-971.

7. Wu BU, Johannes RS, Sun X, Conwell DL, Banks PA. Early changes in blood urea nitrogen predict mortality in acute pancreatitis. Gastroenterology 2009;137:129-135.

8. Singh VK, Wu BU, Bollen TL, et al. Early systemic inflammatory response syndrome is associated with severe acute pancreatitis. Clin Gastroenterol Hepatol 2009;7:1247-1251.

9. Samanta J, Rana A, Dhaka N, et al. Ascites in acute pancreatitis: not a silent bystander. Pancreatology 2019;19:646-652. 


\section{Supplementary material}

Supplementary Table 1 Primary and secondary pancreatitis outcomes based on necrosis or no necrosis

\begin{tabular}{|c|c|c|c|c|c|}
\hline \multirow{2}{*}{$\begin{array}{l}\text { Outcomes } \\
\text { Necrosis }\end{array}$} & \multicolumn{2}{|c|}{ Necrosis $(n=32)$} & \multicolumn{2}{|c|}{ No necrosis $(n=213)$} & \multirow{2}{*}{$\frac{\text { P-value }}{-}$} \\
\hline & - & - & - & - & \\
\hline Extra-pancreatic & - & - & - & - & - \\
\hline Infected necrosis & $15.6 \%$ & $\mathrm{n}=5$ & - & - & $<0.001$ \\
\hline \multicolumn{6}{|l|}{ Mortality } \\
\hline Six-month & $12.5 \%$ & $\mathrm{n}=4$ & $2.4 \%$ & $\mathrm{n}=5$ & 0.004 \\
\hline In-hospital & $15.6 \%$ & $\mathrm{n}=5$ & $3.8 \%$ & $\mathrm{n}=8$ & 0.005 \\
\hline Transient organ failure $^{\dagger}$ & $37.5 \%$ & $\mathrm{n}=12$ & $4.7 \%$ & $\mathrm{n}=10$ & $<0.001$ \\
\hline Cardiac & $15.6 \%$ & $\mathrm{n}=5$ & $2.8 \%$ & $\mathrm{n}=6$ & 0.001 \\
\hline Respiratory & $28.1 \%$ & $\mathrm{n}=9$ & $3.8 \%$ & $\mathrm{n}=8$ & $<0.001$ \\
\hline Renal & $21.9 \%$ & $\mathrm{n}=7$ & $2.4 \%$ & $\mathrm{n}=5$ & $<0.001$ \\
\hline Persistent organ failure $^{\dagger}$ & $37.5 \%$ & $\mathrm{n}=12$ & $3.8 \%$ & $\mathrm{n}=8$ & $<0.001$ \\
\hline Cardiac & $18.8 \%$ & $\mathrm{n}=6$ & $2.4 \%$ & $\mathrm{n}=5$ & $<0.001$ \\
\hline Respiratory & $28.1 \%$ & $\mathrm{n}=9$ & $2.8 \%$ & $\mathrm{n}=6$ & $<0.001$ \\
\hline Renal & $28.1 \%$ & $\mathrm{n}=9$ & $1.9 \%$ & $\mathrm{n}=4$ & $<0.001$ \\
\hline BUN elevation & $37.5 \%$ & $\mathrm{n}=12$ & $20.7 \%$ & $\mathrm{n}=44$ & 0.034 \\
\hline Hematocrit elevation & $68.8 \%$ & $\mathrm{n}=22$ & $25.8 \%$ & $\mathrm{n}=55$ & $<0.001$ \\
\hline \multicolumn{6}{|l|}{ Drainage procedure } \\
\hline Endoscopic & $15.6 \%$ & $\mathrm{n}=5$ & $0.0 \%$ & $\mathrm{n}=0$ & $<0.001$ \\
\hline Radiologic & $12.5 \%$ & $\mathrm{n}=4$ & $0.5 \%$ & $\mathrm{n}=1$ & \\
\hline Surgical & $12.5 \%$ & $\mathrm{n}=4$ & $0.0 \%$ & $\mathrm{n}=0$ & \\
\hline Length of hospital stay (mean \pm SD) & \multicolumn{2}{|c|}{$21.1 \pm 24.2$ days } & \multicolumn{2}{|c|}{$8.0 \pm 15.0$ days } & \\
\hline ICU admission & $46.9 \%$ & $\mathrm{n}=15$ & $9.9 \%$ & $\mathrm{n}=21$ & \\
\hline ER discharge $e^{\ddagger}$ & $0.0 \%$ & $\mathrm{n}=0$ & $15.5 \%$ & $\mathrm{n}=33$ & $<0.001$ \\
\hline Outpatient & $0.0 \%$ & $\mathrm{n}=0$ & $3.8 \%$ & $\mathrm{n}=8$ & 0.025 \\
\hline 30-day re-admission & $15.6 \%$ & $\mathrm{n}=5$ & $19.7 \%$ & $\mathrm{n}=42$ & 0.583 \\
\hline
\end{tabular}

Organ failure defined as failure of one or more organ systems, with failure lasting $>48 \mathrm{~h}$ considered persistent organ failure

${ }^{\ddagger}$ Emergency room (ER) discharge indicates patients who presented to the ER with interstitial edematous pancreatitis but never required admission $B U N$, blood urea nitrogen; $I C U$, intensive care unit; $S D$, standard deviation 